\title{
Citizen Involvement in Service Co-creation in Urban Living Labs
}

\author{
Fumiya Akasaka \\ NTT Service Evolution Laboratories \\ fumiya.akasaka.cx@hco.ntt.co.jp
}

\author{
Momoko Nakatani \\ NTT Service Evolution Laboratories \\ momoko.nakatani.nw@hco.ntt.co.jp
}

\begin{abstract}
Urban Living Lab (ULL) is a living lab in which citizens and companies collaborate to create services for solving problems in a city or region. In ULLs, a variety of citizens participate in a long-term co-creation process including design activities such as concept creation, development, and testing. Unfortunately, few studies have provided useful knowledge about or insights into how to effectively involve citizens with diverse characteristics in such co-creation processes. In this paper, we present a case study illustrating how to involve various citizens in the long-term co-creative design process in ULLs. In this study, we first analyze our ULL project and clarify the various roles that citizens may perform in the co-creation process. Then, on the basis of the analysis results as well as our handson experiences, we provide key insights into obtaining effective citizen involvement in ULLs, which should be helpful to other practitioners and researchers.
\end{abstract}

\section{Introduction}

Co-designing with users is important for developing services or information systems that fit users' needs and promote democratization of processes [1]. Living Lab (LL) is a methodology for designing services with a long-term user involvement approach [24]. In LL, users are actively involved in a co-creative design process in which they collaboratively identify challenges to be overcome, create ideas for responding to the challenges, and test them in the real-life environment of the users [2,5,6]. Besides its "cocreative" characteristic, LL is also considered to be a design approach that has an "in-the-wild" $[7,8]$ characteristic [9], since its design process includes phases for testing and improving services in the real-life environment of the users. LL is attracting global attention as a method for enabling co-created ideas to be implemented in society [10].

As some researchers have suggested, there are various types of LLs with different purposes and design approaches [3,11,12]. For example, some LLs aim to solve social problems in a particular city or region (e.g., [13]) while others aim to develop new technologies (e.g., [14]). We have been conducting an LL project to develop information communication technology (ICT) services for solving local problems in a suburban area of Yokohama, Japan. Our project includes the design activities of "long-term co-creation" and "large-scale social experiments" for ICT service development with citizens. This case corresponds to a type of LL called "Urban Living Lab (ULL)" [15-19] in which a wide variety of local experimental projects of a participatory nature are carried out.

Since ULL is a methodology for solving local problems through long-term citizen participation, citizens with diverse characteristics are involved in various phases of the design process (e.g., problem exploration, idea generation, prototyping, testing); in the process, they have diverse roles. The term "citizen" here does not refer to a general and homogeneous category (group) of people who live in a certain city or area; rather, it refers to a variety of citizens who live in a certain city or area and who have different characteristics [20]. When implementing and operating ULLs, it is therefore important to carefully consider how to involve citizens in the design process while taking into account their diversity. However, few studies have provided useful knowledge about or insights into how to effectively involve citizens with diverse characteristics in the design process in ULL [17].

In this paper, we present a case study that illustrates how citizens with diverse characteristics can be involved into the long-term co-creative design process in ULLs. As argued in previous research on LLs (e.g., [21]), the "role of the actors" is the key to creating new value and achieving innovation in co-creation projects. We therefore focus on the "citizen roles" for better understanding of citizen involvement in ULLs. In this case study, we first analyze our ULL project through the "design reflection" approach [22] and clarify the various roles that citizens may perform in the co-creation process. In addition, on the basis of the analysis results as well as our hands-on experience in the project, we identify key insights into effective citizen involvement. 
The contributions of this paper to the HICSS community are as follows. First, we present a detailed case study of a ULL, which we tried to solve a social problem concerning citizen community activation in a sub suburban area in Yokohama city with the humancentric and in-the-wild design approach. We hope that the detailed case descriptions will inspire further studies or practices of ULLs. Second, we clarify the variation of citizen roles in ULLs. It can be useful knowledge for other practitioners and researchers to manage largescale and long-term citizen involvement for service development. Third, we offer key insights into effective citizen involvement in ULLs. While the insights are derived from a single case study, we believe that they are helpful to other practitioners and researchers when setting up and operating ULLs.

\section{Related studies}

\subsection{Various types of LLs}

Several researchers have described various types of LLs with different purposes, contexts, and design approaches [3,9,11,12]. For example, Leminen et al. [11] proposed differentiating LLs on the basis of which actor drives their activities. Schuurman et al. [12] identified the fourfold categorization of LLs based on a literature review and an empirical investigation of 64 LLs in Europe; the four types are LLs for collaboration and knowledge support activities, original "American" LLs, LLs as extensions to testbeds, and LLs that support context research and co-creation with users. All of these types have in common the same basic characteristics of LLs ("co-creative" and "in-the-wild"), but they differ in their objectives and focused design phases.

Ogonowski et al. [23] pointed out two different LL contexts in the HCI community: controlled and naturalistic settings. Examples of the former context are the MIT PlaceLab [24] and the Aware Home of Georgia Tech [25]. They conducted relatively short-term evaluations in controlled domestic settings resembling typical living spaces equipped with sensors. Examples of the latter context are experiments and data collection related to services and/or technologies that are conducted in the users' real-life environment, not in controlled laboratories [23].

The naturalistic context involves a long-term process including not only experimentation but also cocreation activities with users, in which participatory workshop methods have mostly been used for the cocreation activities (e.g., [26,27]). This long-term cocreation process with users results in a "common understanding" of the problem to be solved [23] and produces deeper and more detailed feedback from users [28]. Alavi et al. [9] conducted a comprehensive survey and analysis of LL-related papers in the HCI field and categorized LLs in terms of the "setup" where design and research were conducted; they identified five setups used in LLs (including both controlled and naturalistic settings).

From another perspective, Hossain et al. [3] argued that the purpose of LLs is also diverse; for example, some LLs aim at technological innovation while others aim at social innovation [3].

\subsection{Urban living labs}

A ULL [15-19] is an LL that focuses on solving local problems and creating urban solutions. ULLs are considered to be an evolution of traditional LLs with which they share the basic characteristics [19]. Although there is no unified definition of ULL, Steen and van Bueren suggested that ULL refers to a wide variety of local experimental projects of a participatory nature that aim at developing, trying out, and testing urban solutions in a real-life context [15]. Thus, ULLs correspond to the naturalistic approach, which includes co-creation and urban experimentation with citizens for the purpose of urban problem solving and social innovation. While it is common in LL studies to use the term "user(s)" to refer to people who use (or will use) the services and/or technologies being created, this study focuses on ULLs, so we use the term "citizen(s)" in a similar sense to "user(s)".

Dalsgaard and Eriksson [29] and Dalsgaard [20] reported on a case study of a large-scale and long-term participatory design project in the city of Aarhus, Denmark. Through this case study, they found that citizens participating in large-scale participatory projects were heterogeneous [20] while normal participatory design projects often involved users with similar attributes. In addition, they found that the difficulty of managing heterogeneous citizens made it difficult to effectively manage participatory projects [20]. Although these works are not ULL studies, they focus on large-scale co-creation processes in a city or region, which means that they are greatly relevant to the context of ULLs.

On the basis of the findings of these previous studies, we can say that the people involved in a ULL project do not belong to a general and homogeneous category (group) of people who live in the city or area; instead, they have various (heterogeneous) characteristics. It is therefore important to carefully consider how to involve citizens with diverse characteristics in the long-term co-creation process when implementing and managing ULLs [18]. However, as noted above, few studies have provided useful knowledge about or insights into how to 
effectively involve such citizens in the design process in ULLs [17].

\subsection{LL user typologies}

Previous LL studies have pointed out the importance of recognizing and managing the diversity of users in LL practice. For example, Ogonowski et al. [23] reported that both technology-savvy and nontechnology-savvy users participated in a long-term LL project and that it was useful to have both types of users involved in the design activities, such as participatory workshops. Leminen et al. described two type of users in LL contexts: active and passive users [30]. While both types should be engaged in LL projects to better achieve innovation [30], Bergvall-Kåreborn et al. observed that getting passive users involved in projects is a challenging task for LL operators [2].

To deeply investigate user involvement in the cocreation process in LLs, several researchers have attempted to identify a typology of "user roles" in LLs [21,30,31]. Nyström et al. [21] proposed four types of user roles: informant, tester, contributor, and co-creator. Informants brings user knowledge and opinions to the LL while testers evaluate services and/or technologies in their real-life environments. Contributors collaborates intensively with the other actors to develop new products, services, processes, and/or technologies. Co-creators co-design a service, product, or process together with the firms' R\&D teams and the other LL actors. These studies on user typologies provide useful insights into how to involve users in the LL co-creation process; however, their usefulness is limited as they do not focus on ULLs, in which there is a greater diversity of users than in LLs.

In the ULL research context, Juujärvi and Pesso [17] investigated "actor" roles in ULLs. While citizens are one of the actors of ULLs, they did not focus on identifying "citizens" roles; they actually referred to the aforementioned four types of user roles (proposed by Nyström et al. [21]) as citizen roles in ULLs. Menny et al. [18] analyzed the different levels of citizen involvement in ULLs but did not explore the specific roles that citizens may perform in the co-creation process in ULLs. Therefore, as noted in these two previous studies, additional investigation is needed to identify the variations in citizen roles in ULLs.

\section{Methodology}

To clarify citizen roles in ULLs and obtain insight into achieving effective citizen involvement in the cocreation process, we adopt a case study research approach (e.g., [32]) in this paper. In this study, we performed two roles of "design practitioners" and "design researchers". We actively participated in a ULL project as practitioners, and at the end of the project period, we analyzed the design process as researchers. In the analysis, we first reflect on and analyze the cocreation process in our ULL project with the focus on the roles that citizens performed in the co-creation process. We then discuss the insights obtained into how to effectively involve various types of citizens on the basis of the analysis results as well as our hands-on experience.

In general, a case study presents results of a unique opportunity to deeply investigate a certain topic [32]. However, since the opportunity is unique, it cannot be completely replicated [29]. Given this caveat, we do not aim to present a specific and universal conclusion that can be applied to all cases or contexts. Rather, we aim to provide findings that, although context-dependent, should be practically helpful to other practitioners and researchers who are now operating ULLs or will be setting up and operating ULLs.

\subsection{Case overview: Tamapla living lab}

The ULL case we address here is the "Tama-plaza Living Lab Project" (hereafter "Tamapla LL"). Tama Plaza is a suburban area in Yokohama city that is convenient in terms of location for commuting to central Tokyo; it is home to many people who wish to live without crowds and noise.

The purpose of Tamapla LL was to develop ICT services that can increase citizen interest in the local area and to encourage community activities by and for locals. The aim is to increase the interest of the citizens in the area in which they live and to motivate them to act in ways that are good for the area. This is an important step in achieving citizen-driven urban development, which is a major goal of the urban development plans for the Tama Plaza area. The project was implemented through the cooperation of local citizens, municipalities, and companies. The companies involved include a railway company that is committed to urban development in the area and a mobile telecommunication company that is developing social ICT services. These companies are two of the largest in Japan; Tamapla LL is thus a valuable case in terms of the proactive participation of large companies. Authors actively participated in the project by planning the cocreative design process and moderating the co-design workshops.

\subsection{Analysis procedure}

We used the "design reflection" approach to investigate the citizen roles and to obtain insights into how to effectively involve citizens. Inspired by the 


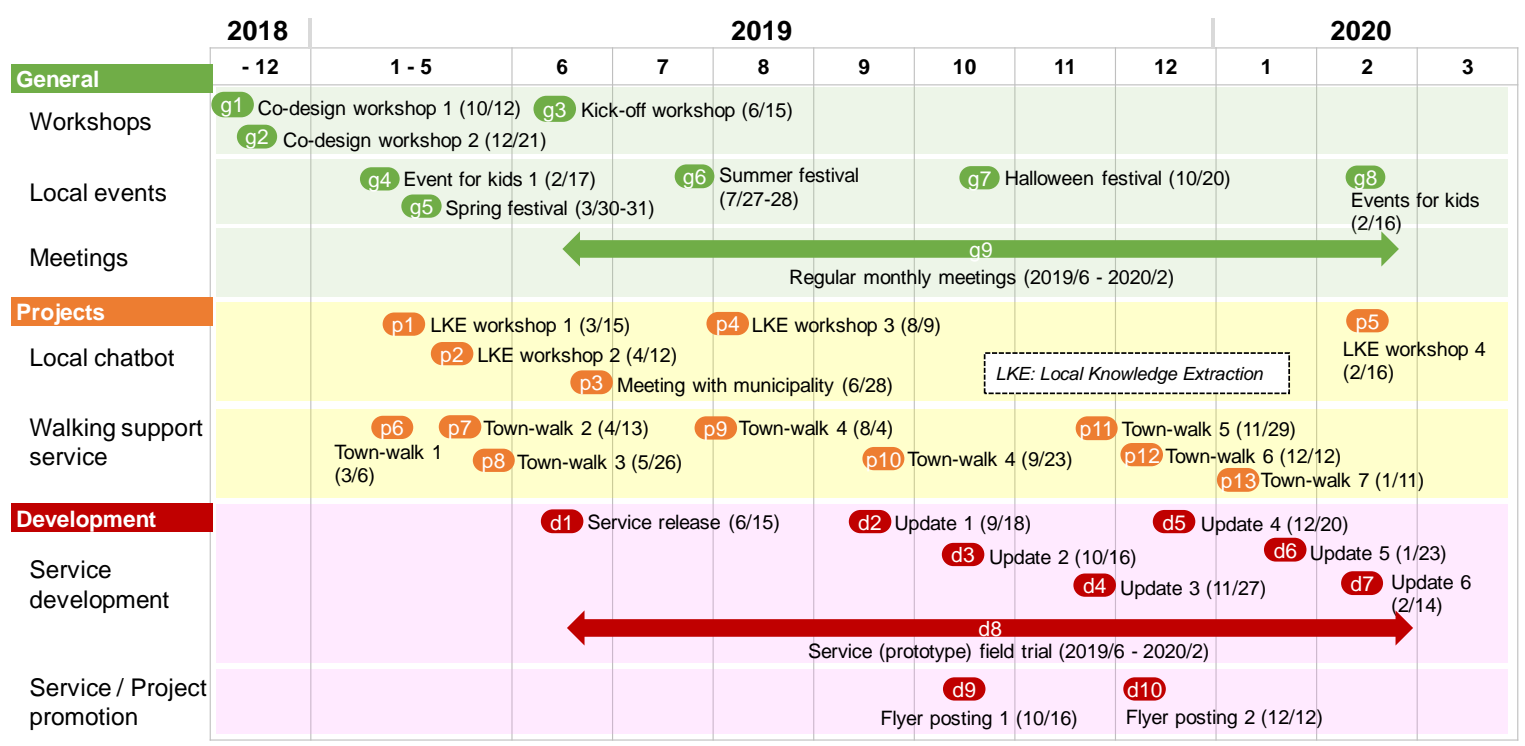

Figure 1. Entire design process in Tamapla LL

reflective design documentation approach [22], we used "activity" as the unit of analysis for the design process. An activity here denotes a design-related activity such as meeting, workshop, experiment, and investigation that were carried out in the ULL project. The design reflection approach adopted in this study allows for a more systematic and detailed analysis of the practiced design process than general participatory approaches.

First, in the design reflection step of the case study, one of the authors and a member, both of them led Tamapla LL, exhaustively extracted information on activities from the data recorded in field notes and communication tools (e.g., e-mails and chat); the extracted activities were then listed in timeline form (see Figure 1). The timings of the service updating were also added to the timeline. Next, we organized a reflection workshop to co-review the timeline with the 17 citizens who actively participated in the project. In the workshop, all the participants including the citizens checked the timeline to correct any omissions in the activities. When missing information was identified, the participants added it to the timeline. We also collected many comments from the citizens on what was good and what could be improved for each activity.

After the workshop, we coded each activity to reflect the perspectives of the roles that the citizens played in the co-creation process. In order to explore and discover new findings from data acquired from practices or observations, it is important to analyze the data by labeling it using "terms" based on the subjectivity of the analyst [33]. Thus, rather than using an existing typology (i.e., the ones described in 2.3), to directly represent what we observed and experienced in our project, we coded the activities using natural language expressed in the form "verb + object" (e.g., co- create service concepts). Then, we summarized them so that the codes with the same meaning were represented by the same linguistic label. The codes were subsequently categorized using an affinity diagram [34] to analyze and identify the variations in citizen roles in ULLs.

In addition to identifying citizen roles, we listed our findings on how to effectively involve citizens on the basis of our hands-on experience as well as comments provided by the citizens in the reflection workshop. We finally summarized the listed findings as design considerations for operating ULLs.

\section{Co-design process research findings}

In this chapter, we describe the detailed design process in Tamapla LL; an overview of the process is illustrated in Figure 1. The project ran for more than one year, from October 2018 to March 2020.

\subsection{Co-design workshops}

The purpose of Tamapla LL was to address local issues in the area. Therefore, we first held a co-design workshop with citizens who were actively promoting local activities to visualize the current problems to be solved and the future visions to be achieved ( $\mathrm{g} 1$ in Figure 1). Two months later, we held a second co-design workshop based on the results of the first workshop to discuss the concepts of the ICT services to be developed in the project $(\mathrm{g} 2)$. As a result of these co-design workshops, we decided to develop (1) a local chatbot and (2) a walking support service as ICT-based services that would be useful for local citizens. 


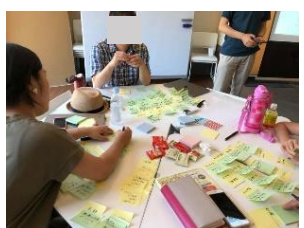

(a) Local knowledge extraction workshop

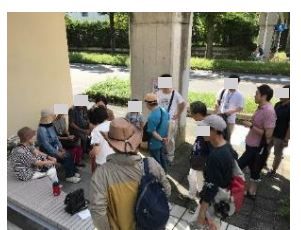

(b) Town-walking event

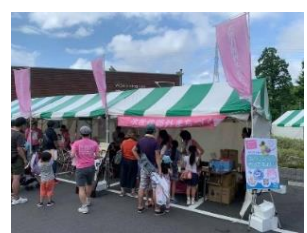

(c) Local summer festival

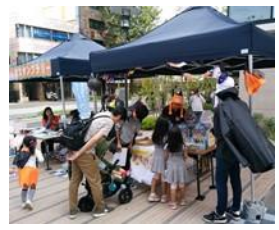

(d) Halloween event

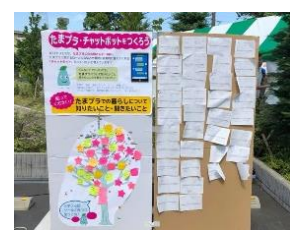

(e) Paper-based questionnaire

Figure 2. Activities with various citizens in the long-term design process in Tamapla LL

\subsection{Service co-development}

The local chatbot is a digital service that provides "local information that only the citizens know" (e.g., good spots for children to play) through the chatbot interface. A local issue addressed by the development of the chatbot was that many citizens, especially ones in their 30s and 40s, were not interested in local activities or resources. This chatbot was aimed at providing an opportunity for many citizens to develop an interest in their local area. The development of the chatbot required the collection of a wide range of local information known by the citizens. We therefore conducted participatory workshops, i.e., "local knowledge extraction (LKE) workshops," to obtain as much local information as possible (Figure 2(a)). We organized four workshops over a period of 11 months (p1,2,4,5 in Figure 1); each one had a specific theme, such as childrearing and food. The local information obtained in the workshops was used as knowledge input for the chatbot.

The target for the walking support service was mainly active seniors in the local area. This service, aimed at increasing citizens' walking activities for their health, has two functions: one is to visualize user's physical activity (e.g., number of steps and calories burned) using wearable activity trackers and the other is to upload and share local attractive spots or recommended walking courses. We collaborated with a citizens' community that was actively organizing townwalking events in the development of this walking support service. The project members in charge of service development participated in each of the townwalk events (Figure 2(b)), which gave them an opportunity to have discussions with the citizens (p6-8 in Figure 1) and to test and improve the service prototypes (p9-13).

Furthermore, during the course of the design process, we had meetings with the citizen leaders once a month. These monthly meetings focused on "how to effectively promote the LL process" and "how to cooperate with important players in the area" (g9 in Figure 1). In addition, we also organized an open event for citizens (i.e., a kick-off workshop, g3) to broadly publicize our LL activities.

\subsection{Social activities}

We actively participated in various "local events;" they were regarded as opportunities to involve citizens in design process (g4-8 in Figure 1). Here, the local event means a large-scale event mostly planned and organized by local citizens, with hundreds or more citizens participating (e.g., local summer festivals shown in Figure 2(c) and Halloween festivals shown in Figure 2(d)). We introduced our LL project and its activities to many citizens and also conducted questionnaire surveys to collect inputs for design. For example, we conducted a paper-based questionnaire survey at the local summer festival (Figure 2(e)) and a smartphone-based questionnaire survey at the Halloween festival in order to collect a variety of data on citizen ideas and opinions, which became input for designing services.

In addition, we designed and distributed promotional flyers to increase the number of service users $(\mathrm{d} 9,10)$. Interestingly, the flyers were also codesigned with citizens who were good at graphic design.

\subsection{Service implementation and evolution}

We implemented the local chatbot ("Tamapla bot") as the main service. An example screenshot of the home screen is shown in Figure 3(a). It was implemented on the instant messaging platform provided by LINE [35], which is the most used text messaging service in Japan. Another service supporting walking activity was developed as a web service (Figure 3(b)) that could be easily accessed from the home screen of the Tamapla bot. The Tamapla bot with the walking support service was released to the citizens in the area in June 2019; then it was tested in the real-life environments of the users as a long-term field trial.

At the time of the initial release of the bot, the number of users was 75 in total; they were mostly participants in the two co-design workshops and kickoff workshop. However, by the end of February 2020, the number had grown to 984 . That is, we were able release our Tamapla bot broadly to local citizens and conduct a large-scale and long-term field trial of the 

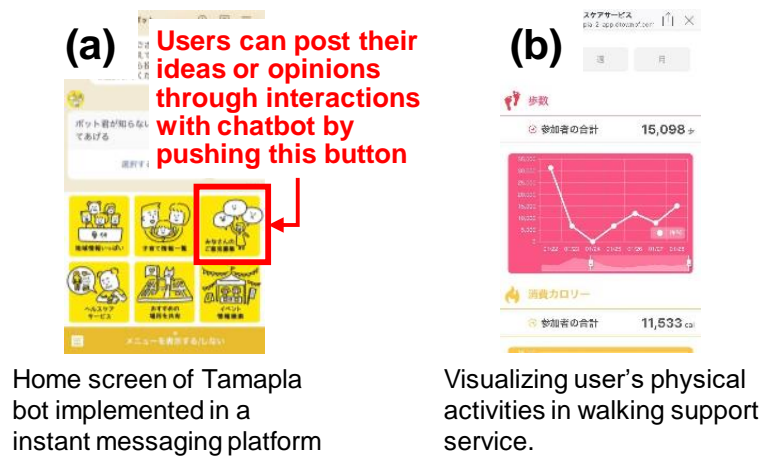

Figuer3. Example of the screenshots of Tamapla bot

services (d8 in Figure 1). We also worked to improve the service during the field trial in addition to testing it. As a result, the bot was updated six times (d1-7) through the design activities described in 4.2 and 4.3.

In the third update ( $\mathrm{d} 3$ in Figure 1), we added a function to the bot that enables users to post opinions, needs, complaints, knowledge, etc. This enables citizens who are users of the services to post their own ideas or opinions anytime and anywhere (Figure 3(a)). Although we basically did not provide incentives for posting, we did provide incentives for a limited period in order to increase contributions from citizens. Since Tamapla LL was a project aimed at revitalizing the local community, we offered discount coupons that could be used at local shops and cafes as incentives for posting.

\section{Diversity of citizen roles}

Through analysis of the design process described in chapter 4, we identified seven citizen roles in ULLs; they are listed in Table 1 and explained below. This knowledge on citizen roles and its categorization can be useful inputs for ULL practitioners to manage or increase the diversity of citizen roles in ULL projects. For example, they can find missing citizen roles in their projects by using this categorization as a checklist.

Informants provide data and information that can be used to design and improve services. There are two types of informant. The first is a person who provides opinions, needs, and/or knowledge. The respondents correspond to this type of informant. The second type is a person who provides information about the local area. These are citizens who are knowledgeable about the local area and provide area-related information (e.g, what people and resources are available in the area).

Concept Creation Partners work together with other actors (e.g., companies) in ULLs to create service concepts. In Tamapla LL, citizens who participated in the early co-design workshops correspond to this role. Since they create service concepts together with other actors from a position of equality, concept creation partners play a very important role that leads to service creation in ULLs.

Development Partners develop or produce information contents that can be used as service components. In Tamapla LL, for example, information contents that were accumulated in the chatbot (i.e., local information known only to the citizens) were extracted jointly with the citizens. This process can be regarded as co-production of service contents. In addition, local citizens designed and created the icon and character images of the Tamapla bot. The role of creating such visual contents is also included in this role.

Creative Generators are citizens who have a creative and activist nature; they proactively propose new ways of service usage that other actors have never considered. For example, in the Tamapla LL, we implemented a function to post attractive spots in our walking support service. The intended purpose of this function was to activate citizens' walking activities by visualizing and sharing attractive spots in the area. However, during the project, one of the citizens proposed using this function to create a "disaster response map," which is a map visualizing locations in the area that would be helpful in times of disaster (e.g., (emergency evacuation area, public phones, vending machines that can provide electricity in an emergency, etc.). This was something we had not considered. Surprisingly, several interested citizens organized an event for creating such a map by using the service; they actively gathered participants by themselves and succeeded in creating a map. Such citizens represent the creative generator role.

Testers conduct tests of the services and/or technologies developed in the project and thereby contribute to their evaluation and improvement. In Tamapla LL, citizens who used and tested the Tamapla bot in their daily life or in the town-walking events played this role.

Meta-design Partners are citizen who cooperate with the "meta-design [36]" of the ULL project. The term meta-design here refers to "the design of the design process and environment". In our project, the citizen leaders participated in our monthly meetings to have discussions on how to proceed with the Tamapla LL project. They played the role of meta-design partner. Note that this meaning of the term "meta-design" is slightly different from that in the literature [36]. Metadesign in this study includes activities to plan how to proceed with the ULL project, for example, what procedures to follow, what kinds of workshops and/or events to conduct, when and where to organize the workshops and/or events, and who to invite to participate in them. Citizens who participate in ULLs are not people who are recruited for research but rather are people who actually live or work in the area $[8,15]$. 
Table 1. Citizen roles in ULL contexts

\begin{tabular}{|l|l|}
\hline \multicolumn{1}{|c|}{ Citizen roles } & \multicolumn{1}{c|}{ Description } \\
\hline Informant & Citizens who (1) provide his/her opinions, needs, and/or knowledge or (2) provide information about the area. \\
\hline Concept Creation Partner & Citizens who work together with other actors in ULL to create service concept. \\
\hline Development Partner & Citizens who develop or produce information / visual contents that can be components of the service. \\
\hline Creative Generator & $\begin{array}{l}\text { Citizens who proactively propose new ways of service usage that other actors have never come up with. They have } \\
\text { creative and activist nature. }\end{array}$ \\
\hline Tester & $\begin{array}{l}\text { Citizens who conduct tests of services developed in the project, and contribute to the evaluation and improvement of } \\
\text { the services. }\end{array}$ \\
\hline Meta-design Partner & $\begin{array}{l}\text { Citizen who cooperate with meta-designing, which means designing the design the design process and environment } \\
\text { in ULL. They also contribute to realize collaborations with important local actors. }\end{array}$ \\
\hline Promotion Partner & $\begin{array}{l}\text { Citizens who support to promote and publicize ULL activities in the area; for example those who create flyers } \\
\text { together or inform ULL activities to their friends or acquaintances. }\end{array}$ \\
\hline
\end{tabular}

Therefore, in order to promote ULLs in local area, it is necessary to take into account the internal mechanisms and human relations of the area [28]. From this perspective, a meta-design partner who deeply understands the local contexts can be regarded as a very important person for operating ULLs.

Promotion Partners promote and publicize ULL activities in the area. In Tamapla LL, the citizens who helped create the flyers or helped promote ULL activities and the Tamapla bot to their friends played this role. This role is important because it can lead to an increase in the number of users and collaborators.

Our practical experience in Tamapla LL revealed these seven citizen roles. Note that all seven roles are not necessarily essential for all ULL cases. Rather, these are the roles that citizens may perform in the co-creation process. In Tamapla LL, citizens often took on multiple roles. This means that citizens participating in a ULL may take on one or more of the seven roles.

\section{Key insights from the case}

We obtained several key insights from our analysis, especially regarding effective citizen involvement. We describe them in detail below. Note that this paper takes a case study approach; our aim is therefore not to clarify completely novel implications for ULL practices but rather to exploratory identify key insights and design considerations based on our ULL case.

\subsection{Various means of citizen involvement}

As described in 2.3., Leminen et al. [30] classified LL users into active and passive users. In Tamapla LL, we also found "active" and "passive" citizens. Active citizens performed roles such as development partner, concept creation partner, creative generator, metadesign partner, and/or promotion partner while passive citizens were mainly testers and/or informants.

One important finding is that it was useful to set up various means of citizen involvement, not only participatory workshops, in order to involve passive citizens. In LL studies so far (e.g., [23,37]), the participatory workshop is one of the most frequently used means to involve citizens in design. It requires, however, intensive discussion among citizens in a faceto-face setting; this kind of activity can impose a high hurdle for participation for passive citizens.

To overcome this difficulty, we prepared easier means to participate for passive citizens. For example, as mentioned in 4.3., we used "local events" as opportunities to involve passive citizen (Figure 2(a, b)). At the local events, we conducted questionnaire surveys to collect a variety of data on citizen ideas and opinions, which became input for designing services. We found that answering to questionnaire was not difficult task for the most of citizens, and in addition, these local events were valuable opportunities to involve various types of citizens. This was because many people living in the area, including some who were not interested in the ULL project, came to the event. Another example of an easier means to participate for passive citizens was "digital participation with smartphones." As mentioned in 4.4., we implemented on the Tamapla bot a function that enables citizens to post their ideas or comments (e.g., opinions, needs, and complaints to the LL project or service itself) anytime and anywhere (Figure 2(e)). In Tamapla LL, citizens actually gave us ideas for improving the Tamapla bot by using the posting function. Such digital participation was a useful means to involve passive citizens. Unlike active citizens, who were deeply involved in service development as design partners, the opinions and evaluations of passive citizens were not biased. Therefore, involving a certain number of passive citizens was effective to collect diverse opinions and honest evaluations on services from citizens' point of view.

\subsection{Motivation for participation}

Another important topic for effective citizen involvement is stimulating their motivation to 
participate. As revealed in previous LL research, providing incentives can stimulate motivation to participate in LL projects (e.g., [38,39]). In this case study, we found that providing incentives was an effective means to increase contributions, especially from passive citizens. In addition, while monetary incentives are often offered in previous LL research, we found that non-monetary incentives are also effective to increase citizens' participation. For example, at the local summer festival where we conducted a questionnaire survey, we gave a plain tote bag to citizens who answered the questionnaire (i.e., "citizens as informants") and they created their original tote bag using crayons and stamps (Figure 4). This was a kind of "experiential" incentives that strongly stimulated the participation motivation of kids as well as parents with small children. In addition, as mentioned in 4.4., we provided incentives to citizens who posted their ideas or opinions using Tamapla bot. The incentives were very effective to stimulate the participation motivation; it resulted in a significant increase in the number of posts.

In contrast, the active citizens participated in many design activities (e.g., participatory workshops and project-related meetings) without any incentives. This indicates that they participated in the design activities on the basis of their intrinsic motivations (e.g., a desire to improve the area in which they lived), in contrast to incentives, which correspond to an external motivation. Furthermore, several active citizens remarked that "we enjoy attending workshops and having conversations with other people". They participated in many activities in Tamapla LL, because they found enjoyment in participating in such activities themselves. This finding indicates that it is crucial, when setting up ULLs, to find citizens who have strong motivations or mindsets to contribute to improving the areas in which they live. Such citizens will likely be active core members of the ULL project.

\subsection{Meta-design with citizens}

As one of the findings regarding effective citizen involvement, we would like to stress the importance of "meta-design partners." As discussed in Section 5, a meta-design partner is a citizen who supports the "design of the design process and environment."

A ULL includes many social practices in a specific area. Therefore, LL practitioners and operators have to consider local resources and human relationships when they plan and operate ULL projects; however, this is not easy for them, since they do not deeply understand the internal mechanism in the area. It is thus important to involve citizens who are familiar with the local context and its internal mechanisms as meta-design partners. For example, in Tamapla LL, we used local events such

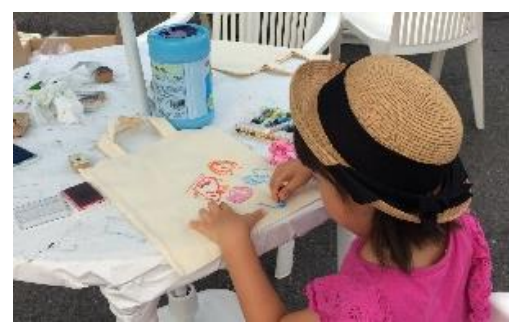

Figure 4. Experiential incentives

as summer and Halloween festivals as opportunities to involve various types of citizens. This idea of using local events for citizen involvement emerged through discussions with citizens who acted as meta-design partners. In addition, when we organized participatory workshops in the early stage of the project, the metadesign partners invited active citizens who were strongly interested in improving their areas or communities as workshop participants. This resulted in obtaining a variety of useful opinions and ideas.

As discussed, we found from our case study that it is important in ULLs to perform meta-designing cooperatively with citizens (especially those who were familiar with local resources and human relationships in the local area) for the effective operation of ULLs.

\section{Discussion and design considerations}

\subsection{Theoretical contributions}

This study contributes to the theoretical discussion of ULLs, especially from the viewpoint of citizen roles. Of the seven roles we identified (Table 1), five (informant, tester, development partner, concept creation partner, and creative generator) were identified in previous research on user roles in LLs (e.g., [21]). The other two roles (meta-design partner and promotion partner) were newly identified in this study. These new roles are more or less related to the meta-design of ULLs, which is crucial for the practice of ULL projects, as discussed in 6.3.

As this study is based on a single case, we do not claim that the seven roles are the complete and definitive typology of citizen roles. However, the seven roles provide an important "foothold" for creating a theory of citizen involvement in ULLs because they were derived from our hands-on experience in a ULL project, one in which we were actually engaged and which we observed for more than a year.

Furthermore, the findings on seven citizen roles can be used not only in the context of services or information systems development, but also policy making and urban planning for considering how to involve citizens in their process. We believe that this 
study is also valuable for researchers and practitioners of citizen-driven urban planning and smart cities.

\subsection{Design considerations}

Below we present five design considerations for practitioners who are about to or will start a ULL project. These considerations were derived from our hands-on experience in Tamapla LL and our in-depth analysis of the project; many of them clearly reflect the key insights explained in Chapter 6. They are our practical contributions to the LL community.

- Use "local events" (e.g., local summer festivals) as opportunities to involve diverse citizens including passive citizens. This kinds of local events are more useful as settings for conducting questionnaire surveys and/or brief service tests than for organizing intensive discussions such as workshops.

- Prepare easier means to participate for passive citizens rather than simply holding only participatory workshops. Use digital technology or devices to enable citizens to participate in design anytime and anywhere.

- Provide incentives to stimulate the participation motivation of passive citizens. Design the contents of incentives in accordance with the citizens' interests or the theme of the ULL project.

- Involve citizens who are highly motivated to improve their area or community. Such citizens are highly likely to become active core citizens in the ULL project.

- Find and recruit citizens who can act as "metadesign partners." Plan how to proceed with the ULL project in cooperation with them. For the planning, have periodic regular meetings with them.

\subsection{Limitations}

Our in-depth analysis of the ULL case in which we engaged enabled us to obtain new findings and key insights that would be difficult to identify with a more objective approach, for example, interview-based investigations of ULL cases implemented by others. However, the findings and insights are derived from a single case; they are not exhaustive and universal conclusions that can be applied to all ULL cases. This limitation derives from the research approach. In future work, we will conduct further ULL practices and expert interviews in order to verify, refine, and update our findings on effective citizen involvement in ULLs.

The design considerations presented in 7.2 are our contributions to practitioners. However, as stated by
Colusso et al. [40], in order to make this kind of knowledge useful for practitioners, it is important to translate it into artifacts (e.g., tools) that are easy for practitioners to apply in their practice. In short, design knowledge presented only in the form of academic papers is not particularly useful for practitioners. Our future research will therefore include the development of tools, such as guidebooks, design cards [41], and design games [42] that will help ULL practitioners plan and operate a long-term co-creation process with citizens with diverse characteristics.

\section{Concluding remarks}

ULL is a human-centric and in-the-wild design approach for creating services and/or technologies that address social issues in cities or regions. Although it has gained global attention in recent years, few studies have yet to provide useful knowledge for its effective implementation and operation.

In this study, on the basis of our practice in Tamapla LL, we clarified potential citizen roles in ULLs. We identified seven roles; two of them are new roles that were not explicitly mentioned in previous reports on LLs. This is a theoretical contribution of this study. Furthermore, we presented key insights into effective citizen involvement in the co-creation activities in ULL contexts. The insights are our practical contribution to future ULL practitioners and operators. As discussed above, this study does not provide universal conclusions that can be applied to all ULL cases; additional practices and investigations will thus be carried out in our future research. However, the findings presented in this paper are derived from in-depth analysis of our long-term hands-on experience in the operation of a ULL; we hope that they help researchers and practitioners who are and will be working in the LL field.

\section{References}

[1] E. Kaasinen, K. Huotari, V. Ikonen, M. Niemelä and P. Näkki. 2012. "Three approaches to co-creating services with users", Advances in the Human Side of Service Engineering, 286-295.

[2] B. Bergvall-Kåreborn and A. Stahlbrost. 2009. "Living Lab: an open and citizen-centric approach for innovation”, Int. J. Innov. \& Reg. Dev., 1(4), 356-370.

[3] M. Hossain, S. Leminen, and M. Westerlundd. 2018. "A systematic review of living lab literature", J. Clean. Prod., 213, 976-988.

[4] D. Schuurman, L. De Marez, and O. Ballon. 2015. "Living Labs: a systematic literature review", In Proc. Open Living Lab Days 2015.

[5] B. Bergvall-Kåreborn, M. Holst, and A. Stahlbrost. 2009. "Concept design with a living lab approach", In Proc. HICSS '09, 1-10. 
[6] A. Følstad. 2008. "Living Labs for innovation and development of information and communication technology: a literature review", E. J. Virtual Org. \& Networks, 10, 99-131.

[7] B. Brown, S. Reeves, and S. Sherwood. 2011. "Into the wild: Challenges and opportunities for field trial methods", In Proc. CHI'11, 1657-1666.

[8] J.M. Carroll, and M.B Rosson. 2013. "Wild at home: The neighborhood as a living laboratory for HCI", ACM Trans. Comput. Hum. Interact. 20(3), 16, 28 pages.

[9] H.S. Alavi, D., Lalanne and Y. Rogers. 2020. The Five Strands of Living Lab. ACM Trans. Comput. Hum. Interact. 27(2), 10, 26 pages.

[10] M. Yasuoka, F. Akasaka, A. Kimura and M. Ihara. 2018. "Living labs as a methodology for service design - An analysis based on cases and discussions from a systems approach viewpoint", In Proc. DESIGN2018, 127-136

[11] S. Leminen, M. Westerlund, and A.G. Nyström. 2012. "Living Labs as Open-Innovation Networks", Tech. Innov. Mgmt. Rev., 2(9), 6-11.

[12] D. Schuurman, D. Mahr, L. De Marez, and P. Ballon, 2013. "A fourfold typology of living labs: An empirical investigation amongst the ENoLL community", Int Conf. Eng., Tech. \& Innov. \& IEEE Int. Tech. Mgmt. Conf., 1-11.

[13] A. Emilson, P.A. Hillgren, and A. Seravalli. 2014. "Designing in the Neighborhood: Beyond (and in the Shadow of) Creative Communities", Making Futures: Marginal Notes on Innovation, Design, and Democracy, Chap. 3, The MIT Press, 35-61.

[14] N. Castelli, C. Ogonowski, T. Jakobi, M. Stein, G. Stevens and V. Wulf. 2017. "What happened in my home?: An end-user development approach for smart home data visualization", In Proc. CHI'17, 853-866.

[15] K. Steen and E. van Bueren. 2017. "The Defining Characteristics of Urban Living Labs", Tech. Innov. Mgmt. Rev., 7(7), 21-33.

[16] Y. Voytenko, K. McCormick, J. Evans and G. Schliwa. 2016. "Urban living labs for sustainability and low carbon cities in Europe: Towards a research agenda", J. Clean. Prod., 123, 45-54.

[17] S. Juujärvi and K. Pesso. 2013. "Actor Roles in an Urban Living Lab: What Can We Learn from Suurpelto, Finland?", Tech. Innov. Mgmt. Rev., 3(11), 22-27.

[18] M. Menny, Y. Voytenko, and K. McCormick. 2018. "Urban living labs and the role of users in co-creation", GAIA, 27(1), 68-77.

[19] G. Nesti. 2018. "Co-production for innovation: The urban living lab experience", Policy \& Soc., 37(3), 310-325.

[20] P. Dalsgaard. 2010. "Challenges of participation in largescale public projects", In Proc. PDC'10, 21-30.

[21] A.G. Nyström, S. Leminen, M. Westerlund and M. Kortelainen. 2014. "Actor roles and role patterns influencing innovation in living labs", Ind. Mktg. Mgmt., 43(3), 483-495.

[22] P. Dalsgaard and K. Halskov. 2012. "Reflective design documentation", In Proc. DIS'12, 428-437.

[23] C. Ogonowski, B. Ley, J. Hess, L. Wan, and V. Wulf. 2013. "Designing for the living room: Long-term user involvement in a Living Lab", In Proc. CHI'13, 15391548.
[24] S.S. Intille, K. Larson, J.S. Beaudin, J. Nawyn, E.M. Tapia and P. Kaushik. 2005. "A living laboratory for the design and evaluation of ubiquitous computing technologies", In Proc. CHI EA’05, 1941-1944.

[25] C.D. Kidd, R. Orr, G.D. Abowd, C.G. Atkeson, I.A. Essa, B. MacIntyre, E. Mynatt, T.E. Starner and W. Newstetter. 1999. "The aware home: A living laboratory for ubiquitous computing research", In Proc. Int. Workshop on Cooperative Buildings, 191-198.

[26] C. DiSalvo, A. Clement and V. Pipek. 2012. "Participatory Design for, with and by communities", Int. Handbook of Participatory Des., Chap.8, Routledge.

[27] Tools for Co-Creation, https://unalab.enoll.org/ (accessed 2020-06-29).

[28] B. Ley, C. Ogonowski, M. Mu, J. Hess, N. Race, D. Randall, M. Rouncefield and V. Wulf. 2015. "At Home with Users: A Comparative View of Living Labs, Interacting with Computers", 27(1), 21-35.

[29] P. Dalsgaard and E. Eriksson. 2013. "Large-scale participation: A case study of a participatory approach to developing a new public library", In Proc. CHI'13, 399408.

[30] S. Leminen, A.G. Nyström and M. Westerlund. 2015. “A typology of creative consumers in living labs", J. Eng. \& Tech. Mgmt., 37, 6-20.

[31] D. Schuurman, K. De Moor, L. De Marez, and T. Evens. 2010. "Investigating user typologies and their relevance within a living lab-research approach for ICTinnovation", In Proc. HICSS'10, 1-10.

[32] B. Flyberrg. 2006. "Five misunderstandings about casestudy research", Qualitative inquiry, 12(2), 219-245.

[33] R. Scupin. 1997. "The KJ method: A technique for analyzing data derived from Japanese ethnology." Hum. Org., 56(2), 233-237.

[34] H. Beyer and K. Holtzblatt. 1997. "Contextual design: defining customer-centered systems". Elsevier.

[35] LINE, https://line.me/en/ (accessed 2020-06-29).

[36] G. Fischer and E. Scharff. 2000. "Meta-design: Design for designers", In Proc. DIS'00, 396-405.

[37] L. Malmborg, E. Grönvall, J. Messeter, T. Raben and K. Werner. 2016. "Mobilizing senior citizens in co-design of mobile technology", Int. J. Mobile HCI, 8(4), 42-67.

[38] A. Georges, D. Schuurman, B. Baccarne, and L. Coorevits. 2015. "User engagement in living lab field trials", Info, 17(4), 26-39.

[39] A. Habibipour, A. Georges, D. Schuurman and B. Bergvall-Kåreborn. 2017. "Drop-out in Living Lab Field Tests: A Contribution to the Definition and the Taxonomy", In Proc. Open Living Lab Days 2017, 7-20.

[40] L. Colusso, C.L. Bennett, G. Hsieh and S.A. Munson. 2017. "Translational resources: Reducing the gap between academic research and HCI practice", In Proc. DIS'17, 957-968.

[41] A. Fedosov, M. Kitazaki, W. Odom and M. Langheinrich. 2019. "Sharing economy design cards", In Proc. CHI'19, $1-14$.

[42] O.S. Iversen and J. Buur. 2002. "Design is a Game: Developing Design Competence in a Game Setting", In Proc. PDC'02, 22-28. 